\title{
EL LÉXICO DE LA MEDICINA: USOS METAFÓRICOS Y METONÍMICOS
}

The lexic of medicin: metaphorical and metonymic uses

\section{Gilberto Castro Delgado*}

\section{RESUMEN}

El objetivo de la siguiente ponencia es hacer una relación del léxico científico de la medicina, especialmente de los términos anatómicos, con procesos normales y cotidianos de la actividad mental humana como la metáfora y la metonimia. En tal relación podrá notarse cómo una expresión lingüística cualquiera resulta en lenguaje figurado por medio de mecanismos de analogía y de correspondencia entre los hechos de mundo y la conceptualización de ideas.

Palabras clave: categorías radiales, uso enciclopédico, lexicón mental, imágenes esquemáticas, experiencialismo, lenguaje figurado.

\begin{abstract}
The aim of this article is to make the relation between medical terminology, anatomical terms, and the common and everyday process of human mental activity such as metaphor and metonymy. In the relation we could see how a linguistic expression becomes figurative language through some mechanisms of conceptualizing the world facts, such as analogy and mapping.
\end{abstract}

Key Words: encyclopedic use, scheme images, mental lexicon, experiencialism, figurative language. 


\section{Introducción}

En estudios llevados a cabo en las últimas décadas por parte de reconocidos lingüistas de las corrientes generativa y cognitiva (Lakoff, Langacker, Talmy, por ejemplo), se ha demostrado que las actividades mentales de los seres humanos obedecen a patrones de experiencia (experiencialismo) entre ellos y la vida cotidiana.

En dichas posiciones teóricas se ha indicado por mucho tiempo que los usos de la metáfora y la metonimia (así como el símil) conforman un mecanismo de asociación de los conceptos con las palabras, y también que no es un proceso pleno o exclusivo del acontecer literario, sino que constituye un proceso cognitivo inherente a la mente humana y que está relacionado con procesos mentales de identificación y conceptualización de los contenidos semánticos en las palabras y en las estructuras gramaticales en una relación analógica con el mundo.

Por lo tanto, el léxico de la terminología médica constituye un campo ingente para aplicar las consideraciones del experiencialismo y del cognitivismo en el análisis etimológico de vocablos científicos médicos y también para detallar en ellos su contenido semántico; su relación entre el latín, el griego y la lengua técnica o científica, así como la correspondencia con la lengua no técnica.

Aspecto fundamental de gran utilidad será repasar la medicina en su correspondencia con la visión por parte de los filósofos de la antigüedad y con distintas etapas históricas de la ciencia médica y la formulación de neologismos en cada una de esas etapas.

Así, se expondrá la relación experiencial de términos como melancolía, histerectomía, útero, pelvis, testículo, vagina, laringe, humor, etc., $\mathrm{y}$, a través del análisis se podrán determinar los usos figurados en la composición de términos como resultado de experiencias de mundo.

\section{Medicina y tradición}

Si bien es cierto que no sólo los griegos y romanos sino también otros pueblos, como los egipcios y babilonios, tuvieron ideas claras sobre la ciencia en general, constituye una enorme tradición los conocimientos aportados por la cultura clásica greco-romana a la cultura occidental.

Resulta innegable que nuestra manera de pensar y de realizarnos en el mundo actual es un reflejo del saber de los antiguos griegos y latinos; eso sí, un saber que se ha visto en la necesidad de actualizaciones en las distintas etapas históricas del mundo occidental. En el campo de la ciencia, también los antiguos demostraron tener un interés y una particular dedicación al conocimiento científico a través de la continuidad en las investigaciones y en lo acertado de sus propuestas hasta el punto de constituir la columna vertebral de la ciencia actual.

Desde los antiguos griegos y romanos, la tradición científica se ha desarrollado a partir del concepto de armonía ${ }^{1}$ que debe prevalecer en el cuerpo humano y en el universo en general. Esta armonía del cuerpo se ve identificada con los conceptos de integridad física y mental, la cual debe constituir un verdadero placer para las relaciones sociales y familiares. Esto nos lleva a la idea de un macrocosmos y un microcosmos: la persona, como entidad física, es reflejo del mundo circundante, de un universo completo, ordenado y armonioso, pero a la vez complejo, y por lo tanto se espera un ordenamiento (cosmos) adecuado en cada ser humano. De ahí la necesidad que tuvieron los médicos, filósofos y sacerdotes de atender y desarrollar una ciencia (o técnica) completa y compleja.

\section{Medicina y filosofía}

De los aportes de la ciencia clásica, la filosofía representa un parámetro esencial para el desenvolvimiento de ese conocimiento general y básico de una cultura cuyo interés científico iba de la mano con la filosofía, ya que los filósofos, en especial los presocráticos, prepararon el camino del conocimiento a los futuros pensadores clásicos, tanto filósofos como científicos. 
Hay que recordar que los presocráticos, especialmente los jonios, fueron los primeros que comenzaron cuestionando su mundo circundante y a ver de una manera distinta el conjunto de conocimientos transmitidos por la literatura (Homero y Hesíodo) y su ámbito mitológico, y de allí surgió la necesidad de plasmar el conjunto de resultados e hipótesis en lo que se denominó la física, o sea, el estudio de la naturaleza en todas sus dimensiones. Por tanto, estos primeros experiencialistas se llamaron físicos ${ }^{2}$ y se caracterizaron por reducir todo efecto a una causa, y a descubrir en la relación causa-efecto la existencia de un orden general y necesario.

Las ideas presocráticas crecieron y se asociaron a nuevas formas de pensamiento en los filósofos llamados clásicos: Sócrates, Platón y Aristóteles. Con estos, la filosofía, sistemáticamente muy madura, alcanzó los vértices de los ideales de una cultura que sabía muy bien desarrollar, homogéneamente, los planteamientos de un ideal de civilización. Y es que los nombres de Galeno, Varrón y Celso no eran nombres de personas cuya profesión era ser únicamente médicos, sino pensadores y estudiosos de las asuntos de la naturaleza.

Pero lo que sí resulta claro es que la medicina griega se convirtió en una técnica consciente y metódica bajo la influencia de la filosofía, principalmente la filosofía jónica de la naturaleza. Por lo tanto, la medicina siguió el camino y la sistematización de la filosofía hasta el Renacimiento, a pesar de haber tenido tantos avances en la composición anatómica, la técnica de los humores, la prevención de enfermedades, etc.

\section{El léxico}

El léxico (Fernández Lagunilla-Anuda Rebollo, 2004) es una especie de diccionario o glosario en el que se especifican las propiedades idiosincráticas de las piezas léxicas de una lengua: pronunciación, significado, categoría gramatical, información semántico-funcional, etc.

Aspecto importante del componente léxico es que los elementos recogidos en este se conciben no como átomos sino como haces o matices de rasgos a través de los cuales se expresan las propiedades inherentes e idiosincráticas de cada pieza léxica, es decir su información gramatical.

Así, en el componente léxico de la gramática se utiliza una nomenclatura que hace referencia al léxico mental, tales como categorías radiales, haces de significado, campos semánticos, redes conceptuales, usos enciclopédicos, continuum de significados y conceptualizaciones.

En el léxico de la medicina resulta común visualizar estas referencias conceptuales que se pueden organizar según diversos patrones de conceptualización. El más generalizado es el de las categorías radiales que, como los campos semánticos, representan expresiones de extensión semántica de los ítemes léxicos a manera de mapeos $^{3}$ o correspondencias cognitivas.

\section{La metáfora}

La expresión lingüística, el lenguaje, es el resultado del pensamiento. Entonces, si la expresión lingüística resulta en lenguaje figurado, es porque la mente se maneja por un mecanismo de analogía y de correspondencia de los hechos y sus denominaciones. La metáfora y la metonimia son un producto de estados mentales.

El pensamiento figurado se da en cada momento de la vida cotidiana y de la manera más normal de lo que se piensa; no es exclusivo de lo poético o de lo retórico. Por lo tanto, se habla con lenguaje figurado ya que este resulta de un proceso cognitivo que impregna el lenguaje y el pensamiento habitual.

Así, la base de la metáfora radica en nuestro sistema conceptual. Es un mecanismo para comprender y expresar situaciones complejas usando conceptos más básicos y conocidos (Lakoff y Johnsson, 2003). Ejemplo de lo cotidiano de la metáfora es la expresión para dar a comprender que algo es muy caro hablando en términos monetarios (CuencaHilfelty, 1999): 


\section{Cuesta un ojo de la cara}

Siguiendo a Lakoff y Johnsson (2003) las metáforas se clasifican en metáforas conceptuales y metáforas de imagen.

Las metáforas conceptuales son esquemas abstractos que sirven para agrupar expresiones metafóricas.

Así, el término corazón ${ }^{4}$ (kardía) expresa una variada gama de sentidos metafóricos, entre ellos:

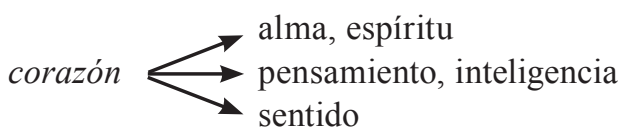

Este tipo de metáfora consiste en la proyección desde un dominio origen hasta un dominio destino.

Se pueden mencionar las siguientes metáforas conceptuales:

$\begin{array}{ll}\text { Radical } & \text { referencia } \\ \text { kolpo- } & \text { vagina } \\ \text { stomacho- } & \text { estómago } \\ \text { gastro- } & \text { estómago } \\ \text { ventri- } & \text { vientre } \\ \text { neumo } & \text { pulmón } \\ \text { sinu- } & \text { seno } \\ & \\ \text { pielo- } & \text { pelvis } \\ \text { torac- } & \text { pecho } \\ \text { pulmón } & \text { pulmón } \\ \text { pelvi- } & \text { pelvis } \\ \text { cisto- } & \text { vejiga }\end{array}$

cavidad-profundidad

griego/latín: significado término médico

\begin{tabular}{|c|c|}
\hline 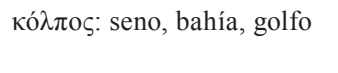 & $\begin{array}{l}\text { colporragia } \\
\text { colpodinia }\end{array}$ \\
\hline 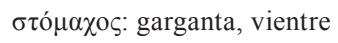 & estomacal \\
\hline$\gamma \alpha \sigma \tau \rho o ́ \varsigma:$ vientre, entrañas & $\begin{array}{l}\text { gastroscopía } \\
\text { gastritis }\end{array}$ \\
\hline venter & vientre \\
\hline$\pi v \varepsilon v \tilde{\mu} o v$ : aire; espíritu & neumococo \\
\hline sinus: cavidad & sinusitis \\
\hline
\end{tabular}

contenedor

\begin{tabular}{|c|c|}
\hline$\pi v ́ \varepsilon \lambda o \varsigma:$ bañera; sarcófago & pielitis \\
\hline v́$\theta \omega ́ \rho \alpha \xi:$ coraza & torácico \\
\hline$\pi v \varepsilon \tilde{v} \mu \mathrm{ov}:$ aire & neumartrosis \\
\hline pelvis: caldero & pelvicectomía \\
\hline 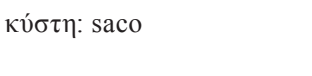 & $\begin{array}{l}\text { cistitis } \\
\text { dacriocisto }\end{array}$ \\
\hline
\end{tabular}

descendencia

$\begin{array}{ll}\text { orqui(d) - } & \text { testículo } \\ \text { histera- } & \text { útero } \\ \text { testículo- } & \text { testículo } \\ \text { genitali- } & \text { genitales }\end{array}$

\begin{tabular}{|c|c|}
\hline ö $\rho \chi \imath \varsigma$ : aceituna & orquidectomía \\
\hline 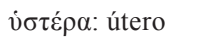 & histeroplasia \\
\hline testis: testigo & testosterona \\
\hline genus: raza & genitourinario \\
\hline
\end{tabular}

flexibilidad- locomoción

$\begin{array}{ll}\text { rotula-, rotela- } & \text { rodilla } \\ \text { cubito- } & \text { codo } \\ \text { humeru- } & \text { hombro } \\ \text { ba- } & \text { caminar }\end{array}$

$\begin{array}{ll}\text { rota: rueda } & \text { rótula } \\ \text { cubitus: codo } & \text { cubital } \\ \text { humerus: hombro } & \text { humeral } \\ \beta \alpha-/ \beta \eta-: \text { ir } & \text { abasia }\end{array}$




\begin{tabular}{|c|c|c|c|}
\hline \multicolumn{4}{|c|}{ protección } \\
\hline matrix- & matriz & matrix: madre & matriz \\
\hline histera,- & útero & 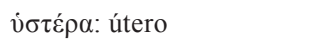 & histerectomía \\
\hline torac- & pecho & v́$\theta \omega ́ \rho \alpha \xi:$ coraza & neumotórax \\
\hline \multicolumn{4}{|c|}{ feminidad } \\
\hline salpingo- & trompas & $\sigma \alpha \dot{\lambda} \pi \imath \gamma \xi:$ trompeta & salpingocele \\
\hline tele- & pezón & $\theta \eta ́ \lambda \eta:$ pezón & distelacia \\
\hline traquelo- & útero & $\tau \rho \alpha ́ \chi \varepsilon \lambda o \varsigma:$ cuello & traquelitis \\
\hline cervic- & útero & cérvix: cuello & cervicitis \\
\hline vagina- & vagina & vagina: vaina & vaginocele \\
\hline ovario- & ovario & ovus: huevo & ovariotomía \\
\hline \multicolumn{4}{|c|}{ interioridad -profusión ${ }^{5}$} \\
\hline entero- & intestino & 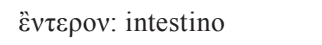 & entrorragia \\
\hline intestino- & intestino & intestinus: interno & intestinal \\
\hline utero- & útero & uterus: útero & uterovesical \\
\hline histera- & útero & 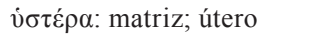 & histerorrexis \\
\hline \multicolumn{4}{|c|}{ lozanía- aprecio- resplandor juvenil } \\
\hline kore- & pupila & кópๆ: niña; muchacha & coreitis \\
\hline pupila- & pupila & $\begin{array}{l}\text { pupa: niña; mucha- } \\
\text { cha }\end{array}$ & pupila \\
\hline pubi- & pubis & pubes: joven & pubertad \\
\hline \multicolumn{4}{|c|}{ inteligencia- ánimo- espíritu de vida ${ }^{6}$} \\
\hline cardia- & corazón & 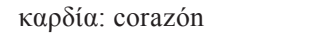 & cardiocele \\
\hline cordi- & corazón & cor, cordis: corazón & cordial \\
\hline fren- & diafragma & $\varphi \rho \eta ́ v:$ entrañas; pericardio & frenitis \\
\hline fronti ${ }^{7}-$ & frente & $\begin{array}{l}\text { frontis: frente; } \\
\text { cabeza }\end{array}$ & frontaomalar \\
\hline neumato- & pulmón & $\pi v \varepsilon \tilde{u} \mu o v:$ aire; espíritu & neumatosis \\
\hline
\end{tabular}

Por su parte, las metáforas de imagen son metáforas concretas que proyectan la estructura esquemática de una imagen sobre la otra (Ungerer-Schmid,1996):

$\begin{array}{llc}\text { Italia } & \text { es una } & \text { bota } \\ \text { destino } & \text { proyección } & \text { origen }\end{array}$

Nótese que la proyección (mapping) no es sino una equivalencia conceptual entre dos dominios. Las metáforas de imagen corresponden a una similitud de la forma: 


$\begin{array}{ll}\text { balano- } & \text { glande } \\ \text { amigdale- } & \text { amígdala } \\ \text { dídimo- } & \text { testículo } \\ \text { peron- } & \text { peroné } \\ \text { colpo- } & \text { vagina } \\ \text { menisco- } & \text { menisco } \\ \text { íleo- } & \text { íleon } \\ \text { pelvi- } & \text { pelvis } \\ \text { pielo- } & \text { pelvis } \\ \text { musculu- } & \text { músculo } \\ \text { mi- } & \text { músculo } \\ \text { clavicula- } & \text { clavícula } \\ \text { cleid- } & \text { clavícula } \\ \text { alveolo- } & \text { alveolo } \\ \text { glande- } & \text { glande } \\ \text { rotula- } & \text { rótula } \\ \text { vesicula- } & \text { vesícula }\end{array}$

\begin{tabular}{|c|c|}
\hline$\beta \alpha ́ \lambda \alpha v o \varsigma:$ bellota & $\begin{array}{l}\text { balanocele } \\
\text { balanopostitis }\end{array}$ \\
\hline$\dot{\alpha} \mu \imath \gamma \delta \alpha ́ \lambda \eta:$ nuez & $\begin{array}{l}\text { amigdalitis } \\
\text { amigdalectomía }\end{array}$ \\
\hline$\delta i \delta \mu o t:$ gemelo & $\begin{array}{l}\text { epidídimo } \\
\text { didimalgia }\end{array}$ \\
\hline broche & peroné \\
\hline 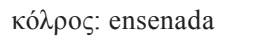 & colpalgia \\
\hline$\mu \eta ́ v:$ luna & menisco \\
\hline 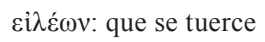 & ileotomía \\
\hline pelvis: caldero & pelvimetría \\
\hline$\pi v ́ \varepsilon \lambda o \varsigma:$ bañera & pielorragia \\
\hline mus: ratón & muscular \\
\hline$\mu \tilde{v} \varsigma:$ ratón & mialgia \\
\hline clavicula: tijereta & clavícula \\
\hline$\kappa \lambda \varepsilon \tilde{\imath} \delta$ os: cerrojo & cleidartritis \\
\hline alvus: cesta & alveolar \\
\hline glandis: bellota & glande \\
\hline rotula: ruedecilla & rótula \\
\hline vas: vasija & vesícula \\
\hline
\end{tabular}

\section{La metonimia}

La metonimia es un tipo de referencia indirecta por la que se alude a una entidad implícita a través de otra explícita.

Si decimos suena el teléfono, hacemos alusión al timbre del teléfono que es lo que realmente produce el sonido en el aparato. $\mathrm{Al}$ decir suena el teléfono nos referimos a la totalidad del aparato por contigüidad. Es decir, en la metonimia se asocian un punto de referencia y una zona activa.

La metonimia se diferencia de la metáfora en que ésta, la metáfora, participa de dos dominios, mientras que aquella sólo participa de un dominio.

El término melancolía se usó en la antigüedad para designar un estado de ánimo en el que intervenía, fisiológicamente, la bilis ( $\chi 0 \lambda \eta ́)$ dentro de una condición de secreción de humores que alteraban el organismo y afectaba el ánimo de las personas. Melancolía significa "bilis negra".

Algunos ejemplos de términos construidos metonímicamente son:

hedrocele: hernia en el recto ( $\% \delta \rho \alpha$ : asiento = lat. sedeo).

atelia: ausencia de pezón en la mujer $(\theta \dot{\lambda} \lambda \eta, \theta \eta=$ mujer).

coxis: hueso de la cadera (coxa).

cara: cara, rostro $(\chi \alpha \dot{\alpha} \alpha \alpha=$ cabeza).

balanopostitis: inflamación del glande y del prepucio $\beta \alpha ́ \lambda \alpha v o \varsigma$; post $=$ más allá).

\section{Conclusiones}

Puede concluirse entonces que, en los pocos ejemplos a los que se ha referido, en la terminología médica el lenguaje figurado de las metáforas y las metonimias se ha adaptado a los procesos cognitivos y de experiencia de mundo a través de usos enciclopédicos, haces de significados y redes conceptuales que los 
investigadores y organizadores han generado en la lengua específica de la medicina en distintas etapas de esta disciplina, y también gracias a la sistematización en la tradición filosóficacientífica en la concepción de la visión de mundo circundante.

\section{Bibliografía}

Bybee, Joan. (2010). Language, Usage and Cognition. New York: Cambridge University Press.

Beristáin, H. (1995). Diccionario de retórica y Poética. México: Editorial Porrúa.

Cárdenas de la Peña, E. (1983). Terminología médica. México: McGraw-Hill Interamericana editores.

Corradi, G. (2005). The Metaphoric process: Connections between language and life. London: Routlege.

Cuenca, M. J., Hilferty, J. (1999). Introducción a la lingüística cognitiva. Barcelona: Editorial Ariel.

Fernández Lagunilla, M., Anula Rebollo A. (2004). Sintaxis y cognición: Introducción a la gramática generativa. Madrid: Editorial Síntesis.

Konstantinidis, G. (2005). Elsevier's Dictionary of Medicine and Biology part $I$. Amsterdam, Netherland: Elsevier.
Lakoff, G. \& Johnson, M. (2003). Metaphors we live by. London: The University of Chicago press.

Lakoff, G. (1990). Women, Fire and Dangerous Things. What Categories reveal About the Mind. Chicago: Chicago University Press.

Langacker, Ronald W. (1987). Foundations of Cognitive Grammar. Vol. I, Theoretical Prerequisites. Stanford, California.:Stanford University Press.

Onysko, A. \& Sascha, M. (2010). Cognitive Perspectives on Word Formation. Berlin: Mouton de Gruyter.

Ortony, A (ed.). (1993). Metaphor and Thought. Cambridge: Cambridge University Press.

Talmy, Leonard. (2003). Toward a Congitive Semantics. Vol. I. Concept Structuring Systems. Massachusetts: Massachusetts Institute of Technology.

Ungerer, F \& Schmid, H. J. (1996). An Introduction to Cognitive Linguistics. New York: Longman.

Van der Eij, P. (2005). Medicine and Philosophy in Classical Antiquity. Union Kingdom: Cambridge University Press.

Uwe Panther, K., Thorngurg, L. y Barcelona, A. (eds.). (2009). Metonymy and Metaphor in Grammar. Netherlans: John Benjamins.

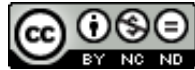

Este obra está bajo una licencia de Creative Commons Reconocimiento-NoComercial-SinObraDerivada 4.0 Internacional. 
